\title{
New Cartesian Generalization of Quantum Mechanics and Relativity Theory Report on the Project "New Cartesian Physics"
}

\section{Boris S Dizhechko*}

Department of Physics, MV Lomonosov Moscow State University, City of Sterlitamak, Bashkortostan, Russia

"Corresponding author: Boris S Dizhechko, Department of Physics, M.V. Lomonosov Moscow State University, City of Sterlitamak, City of Sterlitamak, Bashkortostan, Russia, Tel: 7 4997041341, E-mail: fizika3000@yandex.ru

Received date: August 15, 2017; Accepted date: September 22, 2017; Published date: September 25, 2017

Copyright: (c) 2017 Dizhechko BS. This is an open-access article distributed under the terms of the creative commons attribution license, which permits unrestricted use, distribution, and reproduction in any medium, provided the original author and source are credited.

\section{Introduction}

New Cartesian physics, which is based on identity of space and matter, makes the principle of uncertainty of Heisenberg, from which in quantum mechanics follows a probabilistic way of describing the events, the principle of definiteness of points of space-matter (Principle of physical irrationality). In addition, it considers the rectilinear motion of bodies as a movement on a circle of infinitely large radius that allows it to project the relativistic effects in atom and to relate the probability of events in quantum mechanics with the factor of Lorentz of the special theory of relativity.

In Cartesian identity "space $\equiv$ matter" point of geometric space become material and is therefore able to move [1]. Unlike geometric space, it should be called the physical space, i.e. able to move. The principle of uncertainty of Heisenberg in the physical space turns into its opposite, i.e., becomes the principle of certainty of these points. To show this, take the most known attitude of uncertainty Heisenbergbetween coordinate and momentum of a particle in space:

\section{Where $\hbar$ is Planck's constant (h) divided on $2 \pi$}

Note that here the right and left are the expression of the angular momentum and therefore define an increment radius-vector of rotation, where and the coordinates of the ends of the rotating radius vector $\mathrm{R}$ in physical space [2]. The principle of uncertainty Heisenberg between coordinate and momentum of a particle can then is written as follows:

From this formula we see that when approaching the current coordinates to coordinate the increment of the pulse increases infinitely:

This inequality shows that reducing the interval containing points with coordinates, there is an increase in the increment of the impulse necessary for allocation of these points of space-matter from other points [3]. In the infinitely small interval containing the point, this increment the impulse becomes infinitely large. For this reason, to highlight this point as an independent object impossible. Thus, the uncertainty principle of Heisenberg in new Cartesian physics becomes a principle of physical irrationality of points of space-matter [4].

In mathematics, an irrational number, for example number $\pi$, it is possible to show, with varying degrees of accuracy in intervals:

$$
\begin{aligned}
& 3<\pi<4 ; \\
& 3,1<\pi<3,2 ;
\end{aligned}
$$

$3,14<\pi<3,15$ и т.д.
Similarly, the rational coordinates and that define the interval of localization of the impulse $\mathrm{p}$, determine an irrational point $\mathrm{x}$ is located between them .

Note that here the right and left are the expression of the angular momentum and therefore determines the standard deviation of the radius-vector of rotation $\mathrm{R}$ from the radius-vector $\mathrm{R}_{0}$.

The principle of uncertainty Heisenberg between coordinate and momentum of a particle can be written as:

This inequality shows that reducing the interval containing points with coordinates that correspond to the radius-vectors $\mathrm{R}$ and $\mathrm{R}_{0}$ it is necessary to increase the increment pulse for the selection of this point of space-matter from other points. In the infinitely small interval containing the point, this increment of the momentum becomes infinitely large, which is almost impossible to create [5]. For this reason, to highlight this point as an independent object impossible. It stands out only in the interval. Thus, the uncertainty principle of Heisenberg in new Cartesian physics becomes a principle of physical irrationality of points of space-matter (Figure 1).




Figure 1: Cartesian physics.

Thus, physical objects is not rational points of space-matter which in Euclidean geometry are defined as objects with not have length, not have width, it is irrational points - intervals which however small not were always have length and width.

Intervals that represent the irrational points, and the size of which depends on the acting impulse in isolation, superimposed on each other, are moving. Therefore, to describe the occurrence of the expected configuration of irrational points using methods of probability theory in which a geometrical definition of probability of occurrence of an event [6]. The geometric probability of an event A is the ratio of the measure of area $\mathrm{g}$, is conducive to the emergence of an event A to the measure of the whole area G, i.e. P (A) = mes g / mes G. In this case, the probability of an event is the ratio of the area conducive to its appearance to the entire area of the field where it is expected to be the emergence of $\mathrm{P}(\mathrm{A})=\mathrm{S} / \mathrm{S}_{0}=\mathrm{R} / \mathrm{R}_{0}$ [7].

The sizes of the intervals depend on the size of the current pulse which depends on the speed of movement of the irrational points of physical space. Therefore, the probability of the expected configuration of irrational points depends on the speed of their movement.

In Cartesian physics, it is argued that the movement of physical space, in General, is whirling. In New Cartesian Physics rectilinear motion is represented by motion on a circle of infinitely large diameter or moving at an infinitely small area curved path, i.e. is a part of the whirling motion. If you reduce the radius of this rotational movement of physical space, the speed increases and reaches the speed of light. When this all the relativistic effects associated with motion at a speed close to the speed of light are stored and converted into the parameters of quantum mechanics.

The attitude of uncertainty Heisenberg - between coordinate and momentum of a particle limits existing in the mechanics the law of conservation of momentum:

The law of conservation of angular momentum is formulated for material points possessing mass. According to this law, for a material point (body) with a mass that in the whirlwind of moving in different orbits, will be equality

\section{Reducing in this expression mass, we obtain an expression}

this is Kepler's second law and shows that movement of points of space-matter in the vortex does not depend on mass and applies to its any point. If point of space-matter, revolving around the circumference of the radius vector $\mathrm{R} 1$, sweeps the area $\mathrm{VR} 1$, when reducing the radius of rotation to the value of $\mathrm{R} 0$, the rotation speed will increase and will become equal to $\mathrm{v}=\mathrm{v}_{1}\left(\mathrm{R}_{1} / \mathrm{R}_{0}\right)$.

Let us consider the equality of the angular momentum in an orbit with the velocity $\mathrm{v}$ and the angular momentum in an orbit with the speed of light c:
Consider the equality of the angular momentum on the orbit with speed $\mathrm{v}$ and angular momentum in orbit at the speed of light $\mathrm{c}$ :

Have a few transformations:

Let us denote by as get in final form:

This formula is analogous to the formula for the Lorentz transformation; it shows a reduction of radius at aspiration of speed of rotation to the speed of light. Multiplication on $2 \pi$ the formula is to mind:

, where relevant shows the probability of the fact, that particle will be on the square S0.

Take under consideration in quantum mechanics the wave function:

It can be written as a ratio:

Here A, A1 and A2 - modules of complex numbers, geometrical representation they are radius-vectors. In quantum mechanics the square modulus of the wave function is the probability of finding the particle at the time $t+\Delta t$ in region with coordinates $x+\Delta x$.

By multiplying the numerator and denominator of fraction to $2 \pi$ formulas should be converted to the form:

where relevant indicates the probability that the particle will be on the square $S_{2}$. In this way, reveals the identity of the probability of events in quantum mechanics with the formula of transformation of Lorentz.

According to this expression the probability density of detecting a particle of physical space depends on its speed. If it is at rest in some reference frame, the probability of detecting it is equal to 1 . If it is moving relative to some frame of reference with a speed close to the speed of light, the probability of its detection decreases, which is equivalent to the vacuum of physical space. When moving spacematter at the speed of light, it is on the verge of disappearance and formation on its place the so-called "black hole". When the electromagnetic wave, the formation of black holes is prevented by the movement of space-matter, which manages to fill them with the speed of light. At creation of conditions preventing their completion, such as when whirling motion of a physical space formed independent physical objects, which are called material particles.

\section{References}

1. https://en.wikipedia.org/wiki/Lorentz_factor.

2. Born M (1963) Atomik Physics. London: Glasgow.

3. Descartes R (1998) The World and Other Writings. Trans. Stephen Gaukroger. New York: Cambridge University Press.

4. https://en.wikipedia.org/wiki/Compton_wavelength.

5. Bousso R (2000) Positive vacuum energy and the n-bound. J High Energy Phys 11: Zlatev 1-23.

6. Wang L, Steinhardt PJ (1998) Quintessence, cosmic coincidence and the cosmological constant. Phys Rev Lett 82: 896.

7. http://gsjournal.net/Science-Journals-Papers/Author/180/Boris\%20S., \%20Dizhechko. 